\title{
La marche en crabe de trois types de vraisemblance (empirique, générique et diégétique) dans La Nébuleuse du crabe d'Éric Chevillard
}

\author{
Treveur Petruzziello \\ Université Laval
}

En 1971, Tzvetan Todorov estime que le «concept de vraisemblable n'est plus à la mode » (p. 93). Force est d'admettre que la vraisemblance classique, comme l'entend Aristote dans sa Poétique, dont le rôle « est de dire non pas ce qui a lieu réellement, mais ce qui pourrait avoir lieu dans l'ordre du vraisemblance ou du nécessaire» (p. 65), semble avoir été remplacée par une esthétique du vrai, à laquelle les écrivains contemporains n'attachent visiblement plus autant d'importance que les romanciers réalistes du XIX ${ }^{\text {e }}$ siècle. Ce désintérêt à l'endroit de l'esthétique réaliste avait d'ailleurs 
commencé à se manifester chez les Nouveaux Romanciers. À cet égard, Alain Robbe-Grillet, dans Pour un nouveau roman, précise ceci :

Bien raconter, c'est [...] faire ressembler ce que l'on écrit aux schémas préfabriqués dont les gens ont l'habitude, c'est-à-dire à l'idée toute faite de la réalité. [...] Malheureusement, [...] il s'avère que l'écriture, comme toute forme d'art, est une intervention. Ce qui fait la force du romancier, c'est justement qu'il invente, qu'il invente en toute liberté, sans modèle. Le récit moderne a ceci de remarquable : il affirme de propos délibéré ce caractère, à tel point même que l'invention, l'imagination devient à la limite le sujet du livre. (p. 30)

À la suite des Nouveaux Romanciers, maints écrivains contemporains mettent de l'avant le caractère fictionnel de leur œuvre en plus de bousculer, à des degrés variables, la notion de vraisemblance. La Nébuleuse du crabe, d'Éric Chevillard (1993), couronné du prix Fénéon de la même année, est un cas exemplaire de bouleversement de la vraisemblance, en raison d'une problématisation évidente de trois types de vraisemblance: empirique, générique et diégétique. Corollairement, ce roman échappe à la fois à la cohérence et à la logique du récit attendues, et, forcément, au lecteur. Or, selon Thomas Pavel,

[c]omprendre et apprécier une œuvre de fiction signifie accepter à titre d'hypothèse non empirique l'univers alternatif qu'elle décrit, faire attention aux individus qui l'habitent, prêter foi (provisoirement) aux événements qui s'y déroulent. L'avantage de cette approche [...], [c'est qu'elle] s'intéresse au contenu fictif proprement dit des œuvres littéraires, à la manière dont le texte l'évoque et aux thèses qu'il suggère à propos de l'univers réel. (p. 8)

Autrement dit, même si une œuvre commet nombre d'entorses à la vraisemblance, celle-ci parvient malgré tout à 
formuler une proposition sur le monde. Dans cette optique, notre intérêt ici est d'observer de quelle vision du monde peut participer la Nébuleuse du crabe. Nous avançons que la problématisation de trois types de vraisemblance semble participer d'un projet esthétique. À cet égard, nous prétendons que la dimension empirique, l'appartenance générique et la structure du récit de La Nébuleuse du crabe semblent évoquer la démarche du crabe: le crabe se déplace de côté, donc d'une manière non linéaire. Nous postulons également que cette œuvre propose comme vision du monde une conception de l'homme où une inadéquation assumée avec la société et une marginalité comme mode existentiel sont prônées, et qu'il est possible d'inscrire, à certains égards, cette conception de l'homme dans une perspective rousseauiste. Pour mener à bien cette étude, nous résumerons d'emblée l'œuvre sur laquelle nous nous pencherons et exposerons, par le fait même, de quelle manière La Nébuleuse $d u$ crabe rompt avec la vraisemblance empirique. Nous nous attarderons ensuite sur les entorses aux vraisemblances générique et diégétique, montrant de quelle façon ces transgressions participent d'un projet esthétique. Nous verrons enfin quelles propositions sur le monde peuvent se dégager à la lecture de l'œuvre de Chevillard.

\section{La Nébuleuse du crabe dans la ligne de pêche du lecteur}

Publié en 1993 aux Éditions de Minuit, La Nébuleuse du crabe a été classé dans la catégorie "roman ». Toutefois, cette œuvre est marquée par la brièveté, empruntant à la fois au roman et au recueil de courts textes/fragments sans adhérer 
complètement à l'une ou l'autre de ces formules. Ce roman est en effet divisé en 52 sections, chacune répartie en de multiples fragments délimités par des astérisques. Il s'agit donc d'une œuvre éclatée qui ne présente pas une histoire linéaire que nous pourrions résumer brièvement en en décrivant l'intrigue principale.

Ce roman de Chevillard s'articule autour du personnage protéiforme de Crab qui, à l'image de l'œuvre qui l'accueille, est « "ungraspable", "blurry", and "cloudy" : in a word, nebulous ${ }^{1}$ » (Motte, 2003, p. 45). À cet égard, Crab peut être «mort à la naissance » (Chevillard, p. 36) alors que, le lendemain, il peut naître «avec le cerveau à la place du cœur» (p. 85) et, le surlendemain, «avec [d]es pieds palmés» (p. 102). En fait, comme le souligne à juste titre Olivier Bessard-Banquy dans Le Roman ludique, «Crab paraît n'avoir que son nom d'invariable » (p. 249), contrairement à ses portraits physique, vestimentaire, psychologique et biographique, qui se caractérisent par leur instabilité. Force est donc de constater que Crab rompt avec la conception traditionnelle du personnage, où " caractériser un personnage dans un roman, c'est lui donner, bien que dans la fiction, les attributs de la personne qu'il est censé représenter [...] dans la vie réelle » (Goldenstein, 2005 [1980], p. 52). Du coup, la vraisemblance empirique, qui, selon Andrée Mercier, « concerne précisément [la] conformité de l'univers représenté à l'expérience commune, expérience qui inclut tout autant des connaissances, des faits attestés, que des opinions ou des représentations et qu'on ne saurait donc réduire aux lois physiques et aux données historiques » (2009), est, à l'évidence,

1 Nous traduisons: "insaisissable", "flou" et "trouble": en un mot: nébuleux ». 
largement problématisée dans La Nébuleuse du crabe. C'est plutôt un personnage se réclamant avec force de sa fictionnalisation qui y est présenté.

Ceci dit, le personnage de Crab s'efforce de mener à bien un projet hautement singulier :

Crab a résolu d'opter pour la folie. Non pas sur un coup de tête, détrompez-vous. Les coups de tête n'atteignent que des murs. C'est un projet longtemps caressé, longuement mûri. [...] Le vœu de Crab est de sombrer dans la folie, tête la première, tête la seule, [et] ne garder jouissance que de son corps déboussolé. (Chevillard, p. 12-13)

Pour ce faire, ses entreprises sont multiples : il recoud ses boutons avec un chien (p. 8-9), compte ses cheveux un à un pour mieux se connaître intimement (p. 40), se défenestre immédiatement après avoir terminé de construire sa propre maison (p. 60-61) et s'efforce de « plier le ciel pour le faire tenir dans sa poche » (p. 97). C'est, à n'en pas douter, dans un univers fantaisiste et ludique qu'évolue le personnage de Crab.

\section{Vraisemblances générique et diégétique émiettées, comme de la chair de crabe}

Dans son article «Introduction au vraisemblable», Todorov souligne le caractère polysémique du mot «vraisemblable » et précise qu'une de ses acceptions relève de la vraisemblance générique. À ce sujet, il spécifie que « [l]a liberté du récit est restreinte par les exigences internes du livre lui-même [...], autrement dit, par son appartenance à un genre; si l'œuvre appartenait à un autre genre, les exigences auraient été différentes » (1971, p. 95). De sorte que, à la lecture de $L a$ 
Nébuleuse du crabe, Pierre Jourde ne peut s'empêcher de formuler ces questions : "Cela ne s'appelle-t-il "roman" que par une fiction éditoriale courante? [...] Ce qui émerge dans [...] $C r a b$, est-ce la matière première pouvant servir à composer ici et là tel ou tel roman, et dont les restes reconstitués, accommodés, forment Crab?» (p. 453) Il se trouve que cette œuvre s'écarte visiblement des conventions romanesques entre autres par sa structuration : la fracture en textes brefs crée une discontinuité entre les textes, problématisant, par le fait même, la vraisemblance générique.

$\mathrm{Du}$ reste, le roman crée des attentes en termes de vraisemblance diégétique. Cette vraisemblance, selon Cécile Cavillac, porte "sur la cohérence de la mise en intrigue» (p. 24), c'est-à-dire sur la succession/progression logique des événements du récit. Or, comme le soulève pertinemment Jourde, « [La Nébuleuse du crabe], dont la couverture s'orne du mot "roman", narre bien des événements, mais des événements, si l'on peut dire, purement descriptifs» (p.454). En effet, l'œuvre de Chevillard s'emploie à produire une surenchère de descriptions contradictoires au sujet de Crab plutôt qu'à déployer une intrigue principale. Pourtant, «il existe une structure narrative commune », observe Paul Ricœur, « qui nous autorise à considérer le discours narratif comme un modèle homogène de discours » (p. 3). À cette structure, Jean-Michel Adam donne plus spécifiquement le nom de séquence narrative prototypique et cette dernière est constituée d'une situation initiale, d'une complication, d'une série d'actions, d'une résolution puis d'une situation finale (p.54). C'est dire que, respectant la séquence narrative prototypique, le personnage de Crab devrait être investi d'une quête et son agir devrait être orienté par cet objectif. Cependant, l'agir de Crab semble 
échapper à la fois logique du récit et à la logique de l'action : "Sa ligne de conduite n'apparaît pas très nettement » (Chevillard, p. 8); "Il ne comprenait plus de quoi il retournait, tout à coup, ce qu'il faisait là et surtout ce qu'il devait y faire, pour ne pas décevoir, pour remplir sa fonction, quelle fonction, et comment procéder, et par où commencer, commencer quoi?» (p. 9-10); "Comment occuper ce corps sans rôle qui fonctionne inutilement, que faire de cette tête qui tourne à vide? Il faudrait procurer un travail au premier, des distractions à la seconde. C'est ainsi que Crab passe le plus clair de son temps à se donner des gifles » (p. 63). Crab se singularise donc par un agir désorienté et, du coup, l'œuvre qui l'accueille s'inscrit de manière problématique dans la séquence narrative prototypique. À cet égard, chacun des courts textes qui constituent cette œuvre ne comporte pas nécessairement de complication et de série d'actions. Par conséquent, de la nonmotivation de l'agir de Crab découle une problématisation de la tension narrative ${ }^{2}$, voire une absence de tension. Autrement dit, la question de l'intrigue s'y trouve fortement bouleversée «d'ailleurs, on n'imaginait pas une autre fin que celle-ci pour Crab, la mort étant un phénomène beaucoup trop spectaculaire et bouleversant, [...], l'existence terne et sans intrigue qu'il déroulait ne pouvait légitimement connaître ce dénouement sensationnel » (p. 86; nous soulignons) - et le morcellement en

\footnotetext{
2 Nous empruntons ce terme à Raphaël Baroni, auquel il donne cette définition : «La tension est le phénomène qui survient lorsque l'interprète d'un récit est encouragé à attendre un dénouement, cette attente étant caractérisée par une anticipation teintée d'incertitude qui confère des traits passionnels à l'acte de réception. La tension narrative [est] ainsi considérée comme un effet poétique qui structure le récit et l'on reconnaî[t] en elle l'aspect dynamique ou la "force" de ce que l'on appelle de coutume l'intrigue. » (2007, p. 18)
} 
courts textes contribue, croyons-nous, à fractionner davantage l'agir et l'unité de l'action. Force est de constater que $L a$ Nébuleuse $d u$ crabe s'inscrit en marge à la fois du genre romanesque et des conventions du récit.

\section{La marche en crabe comme projet esthétique}

La Nébuleuse du crabe s'écarte des exigences internes du roman, mais aussi d'une logique recueillistique. L'œuvre, en plus de produire des renversements à l'intérieur même des fragments - ce dont fait montre le premier exemple cidessous - , présente maintes contradictions de court texte en court texte :

Sans l'aide de quiconque. Crab a dessiné les plans de sa maison. Il a choisi ses pierres à la carrière, et il les a taillées. Il a coupé des arbres dans la forêt, pour la charpente. Il s'est muni de matériel. Il a creusé les fondations. Il a trempé son ciment. Il a monté les murs. Il a édifié un escalier sur trois étages. Il a couvert le tout d'un toit. Il a fait les plâtres, la menuiserie. Il a installé la plomberie, l'électricité. Il a collé le papier peint, posé la moquette. Il a meublé chaque pièce selon son goût. Il a gravi l'escalier. Il est entré dans la chambre. Il s'est jeté par la fenêtre (p. 60-61);

- Voici le chantier de ma maison. Je n'ai pas attendu la fin des travaux pour m'y installer, puisqu'elle est habitable, j'ai emménagé hier. Comme vous voyez, le plus gros est fait, ajoute Crab qui désigne pourtant un vaste terrain nu dépourvu de toute trace d'habitation. Mais à ceux qui s'étonnent et tentent de lui démontrer que la construction de sa maison n'a toujours pas commencé, il réplique : - L'entrée et la sortie y sont, toutes les fenêtres aussi, grandes ouvertes, le reste est un luxe dont je me passe très bien. Le Philosophe a son toit dans sa tête, dit-il encore. (p. 71) 
Alors que le lecteur serait en droit de s'attendre à ce que Crab possède une maison nouvellement bâtie, il apprend, dix pages plus loin, que Crab entreprend plutôt de s'en bâtir une sur un terrain laissé vacant. Or, ce revirement de situation, bien qu'étonnant a priori, semble à la fois prévisible et conséquent à la lumière de l'incipit. Le lecteur y apprend que le personnage de Crab se singularise par un esprit de contradiction : «Crab, s'il avait à choisir entre la surdité et la cécité, n'hésiterait pas une seconde et deviendrait sourd sur-le-champ. Pourtant, il met la musique très au-dessus de la peinture. Mais Crab n'est pas à une contradictoire près, on va s'en rendre compte.» (p. 7) L'œuvre, même si elle rompt avec la cohérence et la logique du récit attendues, semble se construire malgré tout avec rigueur et cohésion. Sur ce point, nous avançons que la problématisation des vraisemblances diégétique, générique et empirique participe d'un projet esthétique, soit la marche en crabe de l'œuvre. La non-linéarité du récit, l'inscription de l'œuvre en marge des conventions romanesques et le caractère mouvant de Crab, de concert avec son esprit retors, évoquent la démarche du crabe : ce crustacé marche de côté, c'est-à-dire d'une manière non linéaire, laquelle s'écarte de la norme.

Qui plus est, « le vœu de Crab [qui] est de sombrer dans la folie» (p.13) évoque avec force le désir d'avoir un esprit dérangé/tordu et, plus particulièrement, de porter un regard «oblique» sur le monde, autrement dit de suivre une logique crabienne. Sans compter que le nom de cet être fictionnel fait écho à cette figure animalière et qu'il est également possible d'observer que la graphie tronquée et singulière de son prénom accentue sa marginalité assumée. Crab est manifestement résolu à transgresser les normes et il s'y efforce de diverses manières, dont celle, très révélatrice, de la démarche qu'il 
adopte par un matin où le simple fait d'utiliser son corps pour se mouvoir se révèle un acte auquel il est étranger :

Crab se décida finalement à sortir de chez lui; mais incapable de se rappeler lesquels, d'entre les quatre pieds et mains, étaient réellement appropriés pour la marche, après avoir hésité un instant, il opta pour les mains, plus larges et mieux articulées que les pieds, plutôt que plates, jugeant d'autre part imprudent de trop éloigner du sol sa tête [...]. Crab avait choisi les mains, et lorsque, dans la rue, ayant parcouru sans effort une centaine de mètres, il rencontra ses semblables, leur attitude lui révéla son erreur. Il fit donc comme eux - releva bravement la tête et tomba à genoux. Une épaule pour le joug, une épaule pour la croix - mais Crab se secoua. L'extravagance n'était pas de son côté. Les autres se tenaient mal, pas lui. Ce qu'il avait hâtivement appelé son erreur était bien au contraire un rétablissement magistral, favorisé par la défaillance de sa mémoire et de la confusion de son esprit ce matin-là. (p. 10-11)

Cette façon de marcher qui s'écarte visiblement des conventions et à laquelle Crab croit être le seul à faire honneur fait montre de son excentricité en plus de rappeler, une fois de plus, la démarche oblique du crabe. Cela montre bien que, pour verser dans la folie, Crab emprunte un mode de pensée qui, entraînant des digressions, imite le mouvement caractéristique de ce crustacé : l'esprit de Crab marche donc en crabe. Ces observations nous conduisent à réitérer que les nombreuses entorses aux vraisemblances empirique, générique et diégétique participent d'un projet esthétique, celui de la marche en crabe de l'œuvre. Du reste, Chevillard n'a-t-il pas publié, en 2011, une fiction intitulée Si la main droite de l'écrivain était un crabe, dans laquelle le lecteur peut lire, dès l'incipit, que «Crab ne serait [possiblement] rien d'autre que la main droite d'un écrivain courant latéralement sur la page » (p. 3)? 


\section{La fiction, comme lieu de propositions sur le monde}

À première vue, nous serions tenté de dire que La Nébuleuse $d u$ crabe est un symptôme/produit de la littérature contemporaine en raison, entre autres, de la «décaractérisation» du personnage, de l'éclatement formel et de la problématisation de l'intrigue. Nicolas Xanthos dit d'ailleurs au sujet de l'œuvre romanesque de cet auteur que

c'est bien souvent une littérature au second degré que nous livre Chevillard, une littérature qui questionne en les détournant ses pratiques, ses usages, ses normes, ses valeurs, une littérature qui cherche à se penser ou à se repenser, dans le sillage de Beckett et Michaux, sans doute, mais aussi à sa façon tout à fait singulière. (2009)

Afin de dégager le sens de ce roman, nous serions alors porté à creuser sa valeur ludique, mais «parler de la valeur ludique d'un texte», souligne pertinemment Jourde, "[c']est encore ne rien dire, puisqu'on peut jouer n'importe comment à n'importe quoi, et surtout on peut jouer tout seul » (p. 458). Dans son article «Comment définir la fiction?», Pavel rappelle qu'à

force d'insister sur l'idée que l'œuvre littéraire [...] pose son propre réseau de références, son propre univers, idée exacte jusqu'à un certain point, on en arrive parfois à perdre de vue une des fins les plus importantes de la fiction, qui est de nous exhorter à réfléchir à l'organisation normative et axiologique du monde que nous habitons. (p. 8)

En d'autres termes, même si une œuvre peut faire montre de son caractère fictionnel - lequel est d'ailleurs accentué, dans le cas qui nous intéresse, par les nombreux accrocs à la vraisemblance - il n'en reste pas moins qu'elle formule malgré tout des propositions sur le monde. Ainsi, au lieu de nous 
intéresser exclusivement au monde fictionnel mis en place dans La Nébuleuse du crabe et à ses ruptures avec les usages, il serait bienvenu d'envisager les entorses à la vraisemblance sous l'angle d'éventuelles propositions sur le monde. Nous entendons donc observer de quelle vision du monde peut participer La Nébuleuse du crabe.

Nous postulons que le roman de Chevillard met de l'avant une conception de l'homme où une inadéquation assumée avec la société et une marginalité comme mode existentiel sont prônées. À cet égard, le personnage de Crab serait animé par une quête du devenir-bête, laquelle se décline de deux façons: d'une part, Crab veut devenir bête, c'est-à-dire devenir de plus en plus fou en s'abrutissant/s'abêtissant et, d'autre part, il veut devenir une bête, c'est-à-dire se métamorphoser, d'un point de vue morphologique, en bête. D'ailleurs,

[p]ar le plus grand des hasards, Crab est entré en possession de documents très anciens dont l'authenticité ne fait guère de doute, selon lesquels le millepattes [sic] était un malin, jadis, à l'origine des temps, et l'oie une lumière, et les conférences du buffle attiraient un public nombreux, composé d'abeilles et de linottes, la puce ne buvait pas encore, l'ours était théologien par vocation, le chat cosmographe, l'orang-outan jouait aux échecs, la tortue se mêlait de philosophie, le homard trafiquait en politique... Mais l'évolution se poursuivait, l'adaptation en milieu, chacun de ces animaux vit peu à peu son intelligence diminuer, ses esprits s'obscurcir, sombrer dans sa mémoire et ses facultés raisonnantes, en même temps que se développaient son agilité, sa beauté propre et sa grâce naturelle, ses instincts, sa généreuse santé, dans un monde où la vie s'éprouve par les sens et se conserve par la force. [...] Seul entre tous, l'homme n'est pas parvenu à se débarrasser de sa conscience. (Chevillard, p. 100-101) 
Crab admet alors s'être mis à «envi[er] la bêtise des bêtes, leur vie strictement organique - sans l'horreur des organes - et sensuelle - sans l'effroi des sens - exempte de souci, il aspirait à la folie hagarde, rêvasseuse, du poulpe le plus mou, du lézard le plus plat, de la plus lente des chenilles » (p. 13-14). Cet aveu semble expliquer, partiellement du moins, pourquoi il s'emploie aussi énergiquement à devenir fou. Crab idéalise l'existence animale, laquelle aurait fait, au cours de son évolution, l'expérience de l'intelligence à l'état le plus pur et le plus brut, c'est-à-dire la connaissance naturelle, autrement dit l'instinct. Dans l'extrait précité, le règne animal, après avoir perfectionné ses facultés intellectuelles, a opéré un retour aux sources, à l'état primitif, à ce moment où la bête, divorcée de la raison, agit gouvernée seulement par son instinct. Ce renouement avec l'état primitif pourrait s'expliquer ainsi : l'évolution animale aurait su prouver que la connaissance naturelle supplante la raison, car la bête, pour assurer sa survie et sa défense, et pour tuer ses proies, ne peut se permettre de se perdre dans des considérations philosophiques. Dans cette perspective, Crab élève au rang d'idéal la bêtise et souhaite renouer avec l'état de nature :

Crab s'abrutit jour après jour, trop lentement à son goût, bien sûr, il progresse. À peine rejoint le singe, il songe à rattraper l'âne. Et cet âne n'est qu'une étape. Déjà Crab arrive à la hauteur du phoque. Il a l'autruche en point de mire (p. 101);

Il semble y avoir tant de confort et d'insouciance, de voluptueuse inaction dans la condition de mollusque céphalo, pardon, gastéropode, et si peu de devoirs, de responsabilités, que Crab, quand on le questionne sur ses projets d'avenir, ne cache pas son intention d'opter prochainement pour cet état. L'aveu soulève chaque fois un beau tollé. C'est indigne d'un être humain, s'entend-t-il [sic] répéter. Vous allez baver partout. Si on espère le décourager avec ce genre d'arguments. (p. 112) 
La quête du devenir-bête de Crab semble, à certains égards, s'inscrire dans la lignée de Jean-Jacques Rousseau. La primauté de l'instinct animal sur la raison fait écho aux propos tenus par ce philosophe sur l'homme à l'état de nature dans son essai Discours sur l'origine et les fondements de l'inégalité parmi les hommes. Rousseau y suggère que la société corrompt l'homme et que, pour dépervertir l'homme, ce dernier doit retrouver l'état de nature, un état purement théorique et non politique, dans lequel vivrait l'homme si seulement les lois et le gouvernement n'existaient pas. En d'autres mots, l'homme doit rompre avec la société et la raison, et sa vie originaire doit correspondre à celle de l'animal, qui, lui, est exempt de raison. Ce fondement philosophique découle des réflexions suivantes :

Pourquoi l'homme seul est-il sujet à devenir imbécile? N'est-ce point qu'il retourne ainsi dans son état primitif, et que, tandis que la bête, qui n'a rien acquis et qui n'a rien non plus à perdre, reste toujours avec son instinct, l'homme reperdant par la vieillesse ou d'autres accidents tout ce que sa perfectibilité lui avait fait acquérir, retombe ainsi plus bas que la bête même? Il serait triste pour nous d'être forcés de convenir que cette faculté distinctive, et presque illimitée, est la source de tous les malheurs de l'homme; que c'est elle qui le tire, à force de temps, de cette condition originaire, dans laquelle il coulerait des jours tranquilles et innocents; que c'est elle, qui faisant éclore avec les siècles ses lumières et ses erreurs, ses vices et ses vertus, le rend à la longue tyran de lui-même et de la nature. (Rousseau, 1992 [1971], p. 183-184)

Autrement dit, en prétendant que « l'état de réflexion est un état contre nature et que l'homme qui médite est un animal dépravé»(p.180), Rousseau avance que l'homme, pour se libérer du joug de la société qui tend à dénaturer son être profond, doit répondre à ses «fonctions purement animales : apercevoir et sentir» (p. 189). Dans une telle perspective 
rousseauiste, la quête du devenir-bête de Crab s'inscrirait alors dans une démarche pour recouvrer l'essence de l'homme, mais aussi dans une remise en cause, grosso modo, des fondements de la société.

Cependant, en avançant cela, nous faisons fi de l'un des principaux traits caractéristiques de l'écriture chevillardienne, l'ironie, que Philippe Hamon (1996) considère d'ailleurs comme une forme de l'écriture oblique. Sur ce point, il spécifie que «le discours ironique n'est pas un discours "irréaliste”, ou "nonréaliste", déconnecté de toute référence ou de tout désir de dire le réel. Simplement il le di[t] "de biais", en dehors de tout pacte de croyance fort et autoritaire» (p.65). Cette définition de l'ironie vient consolider notre hypothèse quant à la marche en crabe de l'œuvre. Toutefois, le propre de l'ironie littéraire est de produire des renversements et, plus particulièrement dans le cas de Chevillard, de saboter tout effort interprétatif du lecteur. Alors, en postulant que La Nébuleuse du crabe s'inscrit dans une perspective rousseauiste, nous contribuerons, paradoxalement, à restreindre et à fixer le sens de l'œuvre et, par le fait même, nous prenons part à ce qu'observe ici Bruno Blanckeman :

L'auteur «ne doute pas qu'on va chercher à dégager le sens de son allégorie: elle sera traduite et reformulée en langage simple, en termes clairs et, ce faisant, on le créditera d'ailleurs d'intentions plus nobles que celles qui sont ici revendiquées [...] ». S'en suivent plusieurs phrases qui proposent obligeamment une lecture de la fable de la truite et du choufleur, faisant ainsi de la lecture critique, dans le meilleur des cas, une écaille supplémentaire de la première, dans le pire une circonvolution nouvelle du second. Qui s'aventure à écrire un texte critique [au sujet de l'écriture chevillardienne] commet en cela un délit de fiction, jamais bien loin d'un délire interprétatif. Le théoricien vétilleux des notes de bas de page le notifie sèchement. Il recense les lectures envisageables de son texte, 
coupant l'herbe sous le pied de la critique, et le pied avec, en l'introduisant par anticipation dans la ronde de la fiction, le cercle infernal du sens qui se cherche - dans l'herméneutique du fou. (p. 35-36; la citation est d'Éric Chevillard, L'Auteur et moi)

C'est pour cela que, bien qu'appuyée, l'interprétation que nous suggérons du devoir de folie de Crab doit être perçue comme l'une des lectures possibles, forme de circonvolution que nous nous sommes appropriée précairement, légèrement contaminé peut-être par l'univers délirant du personnage de Crab.

\section{Pour en finir avec la pêche au crabe}

En raison d'une coupure nette d'avec une esthétique réaliste, $L a$ Nébuleuse du crabe se présente comme une œuvre de fiction qui revendique son caractère fictionnel, c'est-à-dire «une représentation littéraire qui constitue un monde autonome, ou du moins partiellement distinct du réel » (Gefen, 2002, p. 216). Plus encore, ce roman de Chevillard, comme nombre d'œuvres contemporaines, s'emploie à bousculer la notion de vraisemblance. À cet égard, le personnage de Crab frappe le lecteur par l'impossibilité de "faire du ciment» (Chevillard, p. 114) avec lui, car « Crab est insaisissable, ni fuyant ni dérobé, plutôt flou » (p. 7). La caractérisation instable de ce personnage l'inscrit en marge de la conception traditionnelle du personnage et, plus spécifiquement, de la vraisemblance empirique. De plus, cette œuvre marquée par la brièveté s'approche à la fois du roman et du recueil de courts textes/fragments sans s'inscrire nettement dans l'un ou l'autre de ces genres, ce qui contribue à fragiliser la vraisemblance générique. L'éclatement formel ainsi 
que les nombreuses incohérences de court texte en texte troublent, pour leur part, la progression logique des événements, bouleversant ainsi la vraisemblance diégétique. S'il est vrai que la problématisation des vraisemblances empirique, générique et diégétique se soustrait à la cohérence et à la logique du récit attendues, il n'en reste pas moins qu'elle semble participer d'un projet esthétique. Sur ce point, nous avons montré que la dimension empirique, l'appartenance générique et la structure du récit de La Nébuleuse du crabe semblent emprunter une démarche similaire à celle du crabe, puisque cette œuvre ne présente ni un récit linéaire ni un récit conforme aux normes établies.

Même si la plupart des critiques prétendent que faire sens avec l'œuvre romanesque de Chevillard est une entreprise vouée à l'échec ${ }^{3}$, nous avons cherché à dépasser le simple constat de ludisme. À la suite de Pavel, nous nous sommes intéressé au contenu fictif au sens strict et avons « consacr[é] toute [notre] attention à la compréhension de ce qui s'y passe » (p. 9). À ce sujet, Alexandre Gefen souligne d'ailleurs que

[c]e qui définit la mimèsis comme la fiction, c'est moins la quête d'une vérité parfaite ou d'une modélisation exhaustive du monde, que la proposition d'un "monde possible" exerçant des liens plus ou moins étroits avec le monde commun et apte, par ce décalage, à fabriquer du sens. (p. 218)

Nous avons alors observé que le devoir de folie de Crab s'inscrit à certains égards dans une perspective rousseauiste, y voyant une façon de résister, bien qu'a priori absurde, à la société. Cependant, le roman de Chevillard, qui produit nombre

\footnotetext{
${ }^{3}$ Lire à cet effet Narjoux et Jollin-Bertocchi (2013) ainsi que Bayard (2014).
} 
de renversements, se joue continuellement de son lecteur et c'est pourquoi le ton ironique de La Nébuleuse du crabe est venu éprouver la solidité de notre observation. La perspective rousseauiste de l'œuvre se présente donc comme l'une des lectures possibles, mais une lecture qui peut, à son tour, être soumise à un regard ironique. Du reste, dans son ouvrage L'Ironie littéraire: essai sur les formes de l'écriture oblique, Hamon note que le personnage du marginal, du vagabond, de l'étranger ou encore de l'intrus est souvent associé à des effets d'ironie (p. 116-117). Ce constat vient très certainement renforcer notre thèse selon laquelle $L a$ Nébuleuse $d u$ crabe exprime une vision du monde où une nouvelle conception de l'homme marquée par son divorce d'avec la société est proposée.

\section{Bibliographie}

ADAM, Jean-Michel. (2008), Les Textes : types et prototypes, Paris, Armand Colin.

ARISTOTE. (1980), Poétique, texte, traduction, notes par Roselyne Dupont-Roc et Jean Lallot, Paris, Seuil, coll. « Poétique ».

BARONI, Raphaël. (2007), La Tension narrative: suspense, curiosité et surprise, Paris, Seuil, coll. « Poétique».

BAYARD, Pierre (dir.). (2014), Pour Éric Chevillard, Paris, Minuit.

BESSARD-BANQUY, Olivier. (2003), Le Roman ludique: Jean Echenoz, Jean-Philippe Toussaint, Éric Chevillard, Paris, Presses universitaires du Septentrion, coll. « Perspectives ».

BlanCKEMAN, Bruno. (2014), "L'herméneutique du fou », dans Pierre BAYARD (dir.), Pour Éric Chevillard, Paris, Minuit, p. 736. 
CaVILlaC, Cécile. (1995), «Vraisemblance pragmatique et autorité fictionnelle », Poétique, no 101, p. 23-46.

Chevillard, Éric. (2006 [1993]), La Nébuleuse du crabe, Paris, Minuit, coll. « Double».

—. (2014 [2011]), Si la main droite de l'écrivain était un crabe, Publie.net.

Gefen, Alexandre. (2002), La Mimèsis, Paris, GF Flammarion, coll. «Corpus Lettres ».

GolDENSTEIN, Jean-Pierre. (2005 [1980]), « Le personnage romanesque », dans Lire le roman, Bruxelles, De Boeck, coll. «Savoirs en pratique », p. 49-72.

HAMON, Philippe. (1996), L'Ironie littéraire : essai sur les formes de l'écriture oblique, Paris, Hachette Supérieur, coll. "Hachette université/Recherches littéraires ».

JouRde, Pierre. (1998), «Crab ou la pêche au gros», dans Valérie-Angélique Deshoulières (dir.), Poétiques de l'indéterminé : le caméléon au propre et au figuré, ClermontFerrand, Association des Publications de la Faculté des Lettres et Sciences Humaines, coll. «Littératures », p. 453467.

MERCIER, Andrée. (2009), «La vraisemblance: état de la question historique et théorique », temps zéro, $\mathrm{n}^{\circ} 2$, <http://tempszero.contemporain.info/document393>.

MotTe, Warren. (2003), «Eric Chevillard's Crab», dans Fables of the novel: French fiction since 1990, Chicago / Normal (Illinois), Dalkey Archive Press, p. 29-49.

NARjoux Cécile et Sophie Jollin-BerTocchi (dir.). (2013), La Langue de Chevillard ou "le grand déménagement du monde», Dijon, Éditions universitaires de Dijon, coll. «Langages» 
PAVEL, Thomas. (2001), "Comment définir la fiction? », dans Alexandre GEFEN et René AUDET (dir.), Frontières de la fiction, Québec/France, Nota bene/Presses universitaires de Bordeaux, coll. « Fabula », p. 3-13.

RICOEUR, Paul. (1980), "Pour une théorie du discours narratif », dans Dorian Tiffeneau (dir.), La Narrativité, Paris, Éditions du C.N.R.S., p. 1-68.

RoBbe-GriLlet, Alain. (1963), «Sur quelques notions périmées ", dans Pour un nouveau roman, Paris, Minuit, p. 25-44.

RouSSEAU, Jean-Jacques. (1992 [1971]), Discours sur l'origine et les fondements de l'inégalité parmi les hommes suivi de Discours sur les sciences et les arts, édition augmentée, présentation, bibliographie et chronologie par Jacques Roger, Paris, GF Flammarion.

Todorov, Tzvetan. (1971), "Introduction au vraisemblable», dans Poétique de la prose, Paris, Seuil, coll. "Poétique», p. 92-99.

XANTHOS, Nicolas. (2009), « Définir Chevillard. L'inconcevable vraisemblance de Démolir Nisard », temps zéro, nㅡ 2, <http://tempszero.contemporain.info/document385>.

\section{Résumé}

En raison d'une problématisation évidente de trois types de vraisemblance (générique, diégétique et empirique), le roman La Nébuleuse du crabe d'Éric Chevillard (1993) échappe à la fois à la cohérence et à la logique du récit attendues, et, forcément, au lecteur. Bien qu'une œuvre commette nombre d'entorses à la vraisemblance, celle-ci parvient malgré tout à formuler une 
proposition sur le monde. Dans cette optique, notre intérêt ici est de dépasser le simple constat de ludisme, en observant de quelle vision du monde peut participer La Nébuleuse du crabe.

\begin{abstract}
Because of obvious problematization three types of plausibility (generic, diegetic and empirical), the novel The Crab Nebula, by Eric Chevillard (1993) lacks both consistency and logic of the expected narrative, and, inevitably, to the reader. Although a number of novels commit breaches of psaubility, it still manages to make a proposal to the world. In this context, our interest here is to go beyond the mere finding of playfulness, observing what vision of the world may participate The Crab Nebula.
\end{abstract}

